\title{
El conflicto. Estudio de caso de una joven tras un proceso de reagrupación familiar
}

\author{
Esther CARMONA PASTOR ${ }^{1}$ \\ carmona.esther@gmail.com \\ Carolina PERAL JIMÉNEZ ${ }^{2}$ \\ carolina.peral@hotmail.com
}

Recibido: 30/06/12

Aceptado: 22/11/12

\section{RESUMEN}

El presente estudio de caso aborda la evolución de una de las participantes del taller de arteterapia "Sentirse en casa" organizado por el Máster de arteterapia UCM/ Grupo de investigación 941035 dentro del proyecto ARIADNE:"Art and adaptation in intercultural enviroments" en el que trabajamos con adolescentes y familias reagrupadas. Entre los participantes del grupo hemos querido destacar el caso de Sandra y, en concreto, un aspecto que ha estado presente durante todo el desarrollo del taller: el conflicto.

Palabras clave: arteterapia grupal, migración, proyecto migratorio, sistemas, reagrupación familiar, conflicto, resistencias.

\section{Referencia normalizada}

Carmona Pastor E., Peral Jiménez, C. (2012). "El conflicto. Estudio de caso de una joven tras un proceso de reagrupación familiar". En Arteterapia: Papeles de arteterapia y educación artística para la inclusión social Vol.7: páginas 153-160. Madrid. Servicio de publicaciones UCM.

\section{SUMARIO}

Metodología para la realización del estudio de caso. Cómo se presenta el caso. Proyecto migratorio. Desarrollo de las sesiones. Conclusiones. Referencias bibliográficas.

\section{Conflict. Case study of a young girl after a family reunification process}

\begin{abstract}
The present study endeavours to describe the evolution of one of the participants in the workshop Feeling at Home organized by the Master's Degree in Art Therapy UCM / 941035 research Group within the ARIADNE project: "Art and adaptation in intercultural environments" with adolescents and families who had gone through a family reunification process.

Over the rest of participants of the group, we want to highlight the case of Sandra and specifically, we want to stress an aspect that has been present throughout the development of the workshop: conflict.
\end{abstract}

Keywords: Art therapy group, migration, migration project, systems, family reunification process, conflict, resistance.

\footnotetext{
${ }^{1}$ Licenciada en Bellas Artes y Arteterapeuta por la UCM. Miembro de AFIA.

${ }^{2}$ Arteterapeuta y licenciada en Bellas Artes por la UCM.
} 


\section{CONTENTS}

Methodology for the implementation of the case study. How the case is presented. Migratory project. Development of the sessions. Conclusions. References.

ESTUDIO DE CASO: SANDRA

Mujer de 18 años

Origen: América del Sur

Lleva en España 2 años

Nombre del proyecto: Sentirse en casa

Vía de intervención principal: arteterapia a través del trabajo plástico

Entidad organizadora: Máster de arteterapia UCM/ Grupo de investigación 941035

Duración del taller: Noviembre del 2011- Abril 2012

\section{METODOLOGÍA PARA LA REALIZACIÓN DEL ESTUDIO DE CASO}

El taller, enmarcado dentro del proyecto Sentirse en casa, ha estado dirigido por dos arteterapeutas en prácticas con dos roles diferenciados, el de observador y el de facilitador. La persona que guía la experiencia puede tener dificultades para atender a todo lo que acontece durante la sesión y esta división en las funciones permitió que la recogida de los datos destacables fuera más efectiva. Como herramienta de recogida de datos se utilizaron la hoja de registro de sesiones, el diario/cuaderno de campo a través de la narración subjetiva, la cámara de fotos y de vídeo.

\section{CÓMO SE PRESENTA EL CASO}

El presente estudio pretende describir la evolución de una de las participantes del taller Sentirse en casa. En concreto, hemos querido destacar un aspecto que ha estado presente durante el desarrollo del taller: el conflicto.

\section{PROYECTO MIGRATORIO}

Sandra, al igual que el resto de participantes, había pasado por una reagrupación familiar. La madre de Sandra llegó a España en busca de una vida mejor, dejando a su hija en su país de origen. Pasados cuatro años y una vez conseguida la residencia legal, pudo reunirse con Sandra en España, quien debió adaptase no solo a un nuevo país, sino también a un nuevo contexto familiar con la actual pareja de su madre y el futuro bebé que planeaban tener en común.

La reagrupación tiene como objetivo volver a unir a los miembros de la familia de las personas migrantes, pero durante este largo proceso, la estructura del núcleo familiar se pierde. Esto da lugar a familias más o menos desestructuradas y con cambios de roles y jerarquías. Así mismo, en los menores se producen pérdidas de vínculos significativos y problemas de integración en el nuevo contexto (nueva estructura familiar, nuevo país, nuevo entorno educativo y amigos, etc.). 
Esta problemática nos hizo plantear una la metodología de abordaje sistémica. Desde el punto de vista de la psicología hay que atender a la idea de que un sistema es un conjunto de elementos que guardan estrecha relación entre sí. Cualquier cambio en uno de sus elementos afecta de una manera indirecta al resto de los elementos que componen el sistema.

En este caso contamos con una usuaria muy joven pues Sandra, apenas tiene 18 años. Partimos de la premisa de que forma parte de múltiples sistemas; familia, clase, grupo de amigos..., y estos grupos no solo determinan su comportamiento sino que están ayudando a la conformación de su identidad.

\section{DESARROLLO DE LAS SESIONES}

Sandra se incorpora al grupo un mes después del inicio del taller. Aparentemente se integra bien dentro del grupo y esto se va a largar durante la siguientes cuatro sesiones. Durante el desarrollo de la primera actividad surge un conflicto personal. Se les pide que dibujen zapatos que simbolicen la fuerza que les impulsa a caminar y los deseos de futuro que quieren conseguir a lo largo de ese camino. Sandra muestra grandes fantasías en sus primeros relatos y escribe que quiere ser actriz, rica, famo$s a \ldots$ al ver cuáles son las expectativas de sus compañeros, menos materialistas, decide cambiarlos por:

Esta bota me ayudará en mi trabajo o en otros más importantes.

Esta zapatilla me ayudará para estar cómoda y tranquila.

Esta zapatilla me ayudará a cumplir mis deseos.
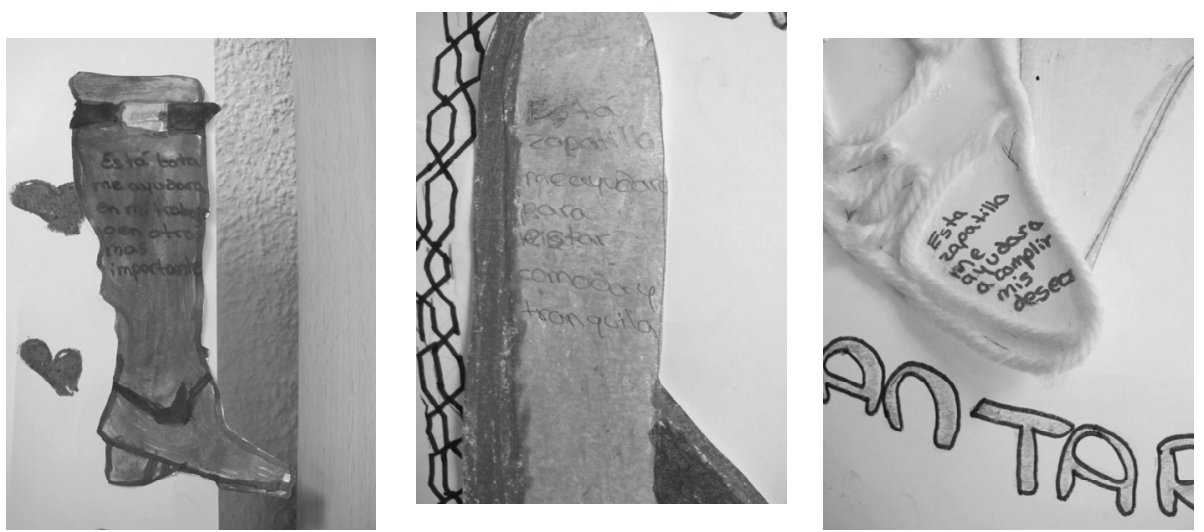

La participante demuestra mucha habilidad para dibujar cuando parte de referentes reales, y despierta la admiración del grupo. Sin embargo, se siente muy perdida en insegura cuando tiene que generar creaciones propias.

Durante el desarrollo de las siguientes sesiones vamos descubriendo aspectos de su personalidad. Es una persona insegura y con una baja autoestima que necesita llamar constantemente la atención del grupo y de las arteterapeutas. En las reflexiones que realizamos durante el cierre de las sesiones, observamos que tiene muy 
poca capacidad de simbolización y no es capaz de relacionar lo surgido en el taller con aspectos personales. En estos momentos se muestra desinteresada, aburrida y con grandes dificultades para escuchar a los demás.

A mediados de Diciembre, en una sesión en la que realizamos una salida a un museo se empieza a ver con gran claridad una fuerte división en el grupo. Cuatro de los participantes se agrupan dejando de lado al resto y Sandra, al observar lo ocurrido, decide aislarse y quedarse fuera por completo. Comenzamos a observar que su manera de enfrentarse a los conflictos es la evitación.

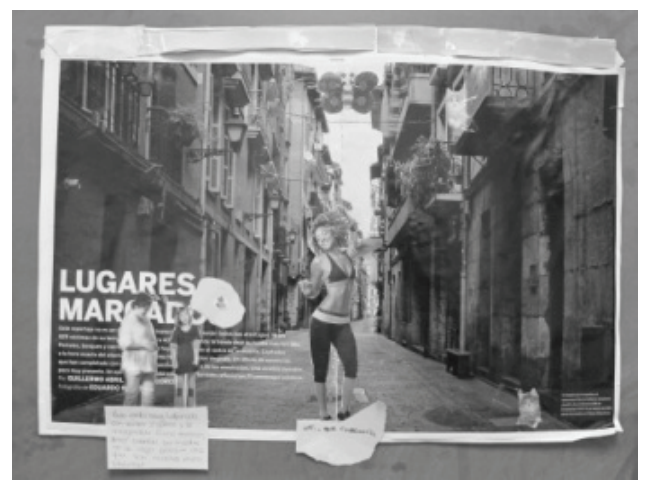

En la siguiente sesión, en la que deben realizar un relato visual en pequeños grupos, se ve que surgen dificultades con Sandra para decidir el tema, como organizarse y realizarlo. En el cierre, durante la exposición de la obra de su grupo manda callar de muy malas formas a la compañera que lo estaba explicando. El conflicto surge y se hace patente que no han podido llegar a un acuerdo durante la realización del trabajo y que, en parte, esto es debido a que ella no cede ante los demás. Cuando le corresponde hablar al resto de grupos, interrumpe sin motivo y se muestra distante y aburrida. Todo esto comienza a generar un gran malestar en sus compañeros.

Sesión tras sesión vamos comprobando que Sandra carece de habilidades sociales, que sus intentos de llamar la atención generan rechazo y que poco a poco la están apartando del grupo, y ella misma también se aísla como mecanismo de defensa.

A finales de Enero, dado que el trabajo con el grupo está muy avanzado, se les pide que ellos mismo sean quienes elijan la técnica y el motivo de su trabajo con una sola premisa, ha de ser un mural en grupo.

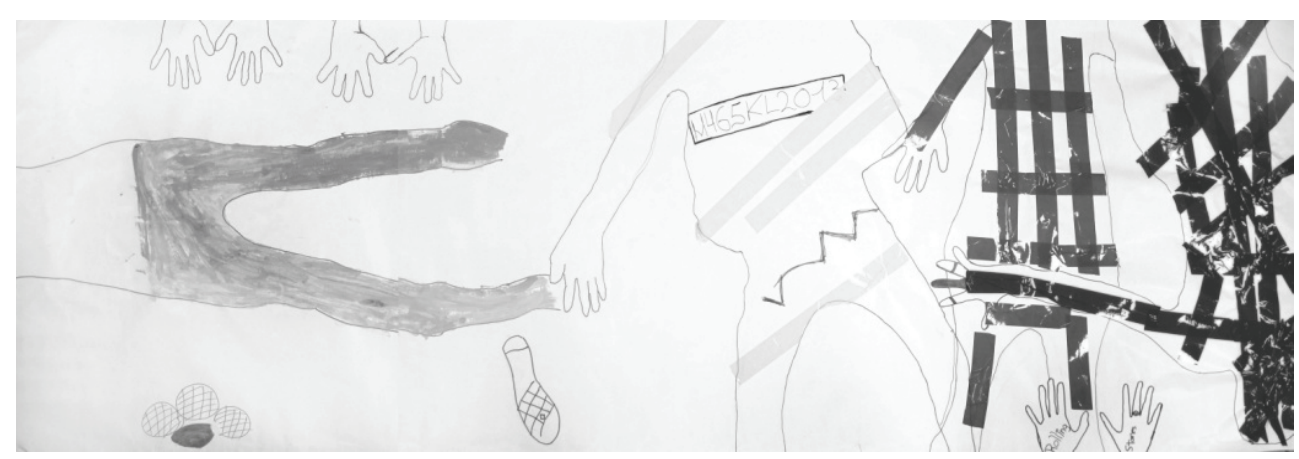

Es curioso que el tema elegido por ellos mismos sea la unión, precisamente algo de lo que carecía el grupo. Durante la realización del mural vuelve a haber 
pequeños conflictos que se hacen visibles en el resultado final de la obra, ya que se ve el cuerpo de Sandra apartado del resto. Además de la separación física y formal, la técnica y el estilo utilizado hacen que quede patente que no consiguen representar la unión, sino la exclusión del grupo hacia esta persona. En el cierre de la sesión se refleja este aspecto del trabajo y el grupo asegura no haberse dado cuenta durante el proceso. La primera reacción de Sandra en el cierre fue la evitación, no quería abordar lo que había sucedido durante la sesión, pero poco a poco al ver que esta apoyada por las facilitadoras se decide a hablar y contar que está harta. Tras esta afirmación se le pide que dibuje un vaso y lo vaya llenando con las cosas que le hacen estar harta. Sus compañeros de estudios, su familia, sus amigos y los problemas del taller... todo ello le hace estar harta. Vemos que su manera de relacionarse en el taller no difiere de la de otros entornos o sistemas de los que Sandra forma parte y que en todas estas situaciones, como nos cuenta ella misma, su manera de manejarlo es dejarlo pasar, no hacer frente.

Aquí pudimos comprobar como las habilidades sociales de Sandra son muy escasas. Cuenta con pocos recursos personales para afrontar sus problemas de vinculación con los demás, y esto le hace estar excluida de todos los grupos a los que pertenece. Nuestra intervención estuvo orientada a ayudarla a buscar nuevas estrategias de afrontamiento ante los problemas. Reflexionamos sobre las reacciones y modos de actuar que creaban rechazo en los demás y sobre su excesiva preocupación sobre lo que piensan los demás de ella. Ella misma llegó a la conclusión de que debía procurar estar satisfecha con su actos y estar más segura de las decisiones que toma ya que no va a poder agradar a todo el mundo.

A pesar de todo lo trabajado durante esta sesión no se ve cambios significativos en su conducta en las siguientes sesiones pero se comprueba que ahora se siente respaldada por las arteterapeutas y esto la hace sentirse fuerte ante el grupo. Su comportamiento se vuelve muy inflexible y autoritario, llegando incluso a faltar al respeto a sus compañeros .Además no se ve una elaboración simbólica en el trabajo de Sandra.

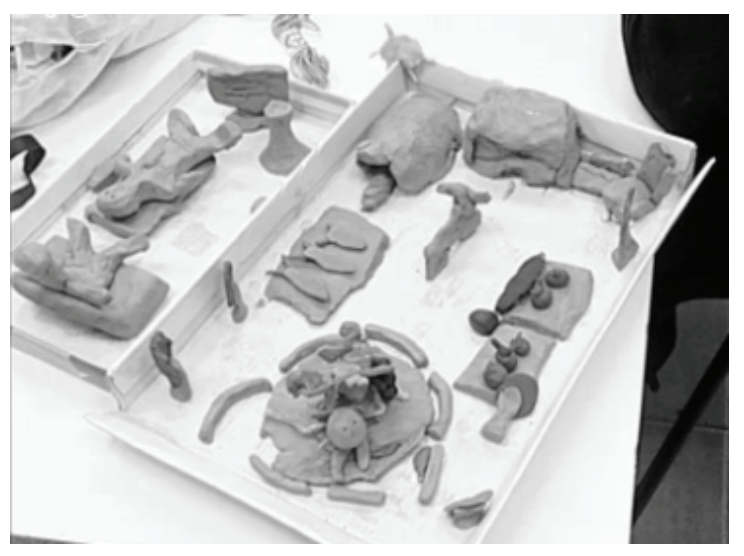

Tras un nuevo trabajo en grupo, la creación de una ciudad imaginaria, vuelve a surgir conflicto. En esta ocasión a pesar de decidir entre todos cuales van a ser las reglas y normas, ella decide tomar la suyas propias. Se excusa diciendo que no han tenido en cuenta su opinión. Aquí el grupo reacciona atacando ya que si en un primer momento asumieron su parte de culpa en los conflictos del taller, en este caso indicaron que ella también 
debía asumir sus errores. El haber estado aparte o excluida había sido por decisión propia para no seguir lo acordado por el grupo y que debía de ser responsable y asumir que esta ocasión ella se había apartado. Vemos como la evitación sigue siendo su estrategia.

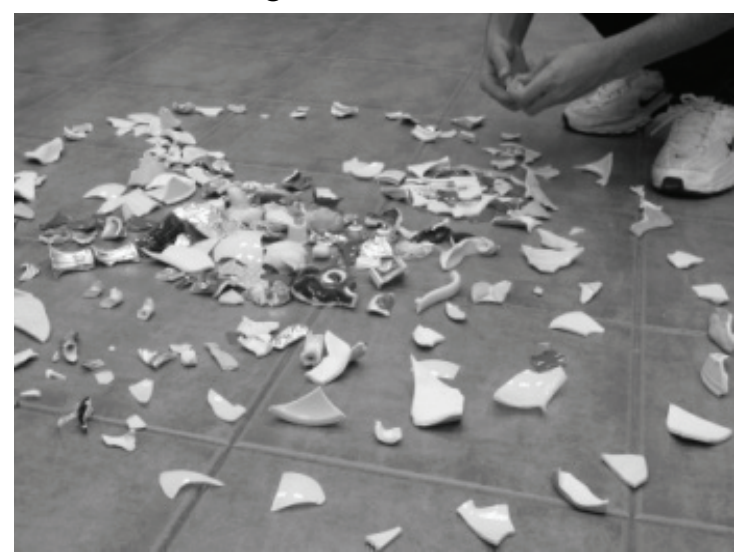

Ya solo quedan dos sesiones para finalizar pero el trascurso de esta última hace plantearse si Sandra debe seguir trabajando con el grupo o si sería más conveniente trabajar de manera individual con ella, ya que la ausencia de cambios y los continuos conflictos hacen a otros participantes querer abandonar el taller. Vemos que el grupo no sale enriquecido de los confictos, ni permite avanzar a Sandra. Su poca habilidad para relacionarse y su baja autoestima son características que hacen pensar que la mejor intervención sería de manera individual pero finalmente, ya que el taller estaba a punto de finalizar, decidimos no variar el modelo de intervención.

En esta época, tenemos conocimiento de que la madre de Sandra ha vuelto a su país de origen a buscar trabajo ante la difícil situación económica de España. Sandra se encuentra sola con la pareja de su madre y el bebé de ambos.

Durante las dos últimas sesiones, de nuevo está presente el conflicto. En la penúltima planteamos realizar un quipu de forma individual que simbolizara el tiempo del taller. Sandra tiene dificultades para entender la dinámica por la necesidad de simbolización y se bloquea. En lugar de pedir ayuda, Sandra se aparta y realiza un trabajo con un valor meramente estético. Ante la propuesta de relacionar y unir los quipus de todos, el grupo la invita a unirse, pero ella declara que prefiere unirse con aquellos que ya no estaban en el taller, lo que provoca el malestar de los demás y un reproche en cuanto a su manera de apartarse.

En la última sesión es un conflicto personal el que aflora. Ante la propuesta de romper con un martillo diferentes figuras de porcelana para crear un mandala entre todos, Sandra se niega, y dice no entender lo que se pide porque las figuras son muy bonitas. Intentamos explicarle que le proponíamos un proceso de trasformación de unas figuras y platos que no tenían valor emocional para ellos, para convertirlo en algo con significación para todos. Sin embargo, lo entiende como un proceso de destrucción y decide no utilizar el martillo. El resto del grupo toma la propuesta como un acto divertido en el que disfrutar del proceso liberador de la destrucción previa a la creación y todos participan en la rotura de las piezas, respetando la opción de Sandra. Una vez que las figuras estuvieron rotas, sí accedió a crear el mandala con el resto del grupo. En el cierre, reflexionamos sobre los procesos de transformación y cambio que ellos mismos habían sufrido en el taller, y de cómo a 
veces nos cuesta mucho trabajo romper con lo conocido y nuestras maneras habituales de comportarnos, aunque nos hagan daño. En este último día también les pedimos que escribieran algo acerca del taller. Estas fueron las palabras de Sandra:

Yo me quedo con lo mejor del taller, no cambiaría nada, porque está todo completo. Espero que algun otro dia haiga otro taller como este o parecido.

Las actividades me han gustado mucho. Depende de algunos dias que estado con mala gana de no hacer nada. Y con el grupo pues me ha gustado estar y compartir con ellos, aunque con algunos menos. Espero que repitan.

\section{CONCLUSIONES}

El caso de Sandra viene a ejemplificar como los conflictos personales y grupales se hacen presentes en las sesiones y son un eje fundamental del proceso terapéutico.

Su participación en el taller ha sido muy positiva para poder ofrecer un espacio en el que abordar y prestar atención a estos conflictos, que están también presentes en otros entornos de Sandra, pero que no son abordados por la propia evitación de la participante. El taller ha permitido a Sandra poner de manifiesto como los conflictos surgidos con ella misma y los problemas de vinculación con los demás, son el reflejo de una problemática que debe ser abordada en profundidad y de forma individualizada, con un ritmo distinto y donde la simbolización no fuera un factor fundamental.

En el caso presentado el tiempo fue un factor determinante. Por un lado, si el final del taller no hubiera estado planteado habría que haber tomado decisiones, pues el conflicto es enriquecedor si todos los miembros que participan de él salen fortalecidos. En este caso, los conflictos personales de Sandra no podían ser abordados en grupo, ya que sus recursos personales, a pesar de haber pasado por una trayectoria similar, no eran los mismos. Por otro lado, su paso por el taller ha supuesto una toma de conciencia de su problemática, y sus pequeños avances y resistencias nos muestran que hubo progresos, pero faltó tiempo. El fin de su terapia no debería de haber sido una imposición, sino la evolución del trabajo.

\section{REFERENCIAS BIBLIOGRÁFICAS}

ACHOTEGUI, J. (2004): Emigrar en situación extrema: el Síndrome del inmigrante con estrés crónico y múltiple (Síndrome de Ulises). Norte de salud mental. N $\mathrm{N}^{\circ} 21$ Pág. 39-52

AGUIRRE BAZTAN, Á. (1994): Psicología de la adolescencia. Barcelona, Marcombo, Boixareu Editores.

BOTELLA, L Y VILAREGUT, A. (s/f). La perspectiva sistémica en terapia familiar: Conceptos básicos, investigación y evolución. Facultat de Psicologia i Ciències de l'Educació Blanquerna Universitat Ramon Llull. Extraído el 22-4-12 de http://jmonzo.net/blogeps/terapiafamiliarsistemica.pdf 
GRINBERG, León y Rebeca (1998): Identidad y Cambio. Barcelona: Paidós Iberica.

GRINBERG, Rebeca (1998): Psicoanálisis de La Migración y del Exilio. Madrid: Alianza.

LÓPEZ FDZ. CAO, Marián y MARTÍNEZ DÍEZ, Noemí (2006): Arteterapia Conocimiento interior a través de la expresión artística. Madrid: ediciones tutor.

MAALOUF, Amin (2007): Identidades asesinas. Madrid: alianza. 